\title{
Mesure de l'activité antiradicalaire du jus et des peaux d'oranges tunisiennes par le radical DPPH
}

\author{
Jamilla Kalthoum CHERIF ${ }^{a *}$, Ines M'RABET ${ }^{b, c}$, Mourad EL HABIRI ${ }^{b}$, Rym ABIDI $^{c}$, Anne-Marie ALBRECHT-GARY ${ }^{b}$
}

a Institut préparatoire aux études d'ingénieurs de Tunis, 1008 Monfleury, Tunis, Tunisie

cherif-j2000@yahoo.fr

${ }^{\mathrm{b}}$ Faculté des sciences de Bizerte, Université du 7 novembre à Carthage, 7021 Jarzouna-Bizerte, Tunisie

${ }^{c}$ École Chimie Polymère Matériaux, Laboratoire de physico-chimie bioinorganique, UMR 7509 CNRS, 25, rue Becquerel, 67200 Strasbourg, France

* Correspondance et tirés à part

Reçu le 16 décembre 2004 Accepté le 29 octobre 2005

Fruits, 2006, vol. 61, p. 99-107 (c) 2006 Cirad/EDP Sciences All rights reserved DOI: $10.1051 /$ fruits:2006008 www.edpsciences.org

RESUMEN EsPaÑoL, p. 107

\section{Measurement of the antiradical efficiency of Tunisian orange juice and skins using the DPPH radical.}

Abstract - Introduction. Orange (Citrus sinensis (L.) Osbeck) is a source of bioactive compounds: $\mathrm{C}$ and $\mathrm{E}$ vitamins, and flavonoids. To develop waste of orange skins, we measured juice and dry and fresh skin antiradical activity of five Tunisian orange tree varieties to assess their concentration in antioxidant potential usable in industry. Materials and methods. The antiradical capacity of juice and skin extracts was evaluated while following, according to time, the disappearance of the $\mathrm{DPPH}^{\bullet}$ stable radical by spectrophotometry of absorption visible-UV at $515 \mathrm{~nm}$. The antiradical activity measured was expressed in effective concentration $\left(\mathrm{CE}_{50}\right)$. The determination of the vitamin $\mathrm{C} \mathrm{V}_{50}$ volume of an ascorbic acid solution with a known concentration and of a juice extract and dry and fresh orange skin $\mathrm{V}_{50}$ volume made it possible to consider the molar and mass concentration of equivalent vitamin $\mathrm{C}$ in the extracts. Results. Orange juices contain approximately $400 \mathrm{mg} \cdot \mathrm{L}^{-1}$ of equivalent vitamin $\mathrm{C}$. In the case of very fast reactions with radical $\mathrm{DPPH}^{\bullet}$, the totality of the antiradical activity can be allotted to the vitamin C. In the case of slower reactions, in addition to the vitamin C, polyphenols (citroflavonoids) contribute to the total antioxidant activity. The fresh skin methanolic extracts contain more vitamin $\mathrm{C}$ than the dry skin extracts. Conclusion. Orange skins constitute immense sources of antioxidants which can find applications in various fields: agro-industrial, cosmetic and pharmaceutical, among others.

Tunisia / Citrus sinensis / fruits / orange juice / peel / waste utilization / natural antioxidants / ascorbic acid

\begin{abstract}
Mesure de l'activité antiradicalaire du jus et des peaux d'oranges tunisiennes par le radical DPPH.

Résumé - Introduction. L'orange (Citrus aurantium sinensis) est une source de composés bioactifs : vitamines $\mathrm{C}$ et $\mathrm{E}$, flavonoïdes (flavanes et flavanones glycosylés et polyméthoxylés). Pour valoriser les déchets des peaux d'orange, nous avons mesuré l'activité antiradicalaire du jus et des peaux sèches et fraîches de cinq variétés d'orangers de Tunisie afin d'évaluer leur concentration en composés antioxydants utilisables dans l'industrie. Matériel et méthodes. Le pouvoir antiradicalaire du jus et des extraits des peaux a été évalué en suivant, en fonction du temps, la disparition du radical stable $\mathrm{DPPH}^{\bullet}$ par spectrophotométrie d'absorption UV-visible à $515 \mathrm{~nm}$. L'activité antiradicalaire mesurée a été exprimée en concentration efficace $\left(\mathrm{CE}_{50}\right)$. La détermination du volume $\mathrm{V}_{50}$ de vitamine $\mathrm{C}$ d'une solution d'acide ascorbique de concentration connue et d'un volume $\mathrm{V}_{50}$ des extraits de jus et des peaux sèches et fraîches d'orange a permis d'estimer la concentration molaire et massique de vitamine $\mathrm{C}$ équivalente dans les extraits. Résultats. Les jus d'orange contiennent environ $400 \mathrm{mg} \cdot \mathrm{L}^{-1}$ d'équivalent de vitamine C. Dans le cas de réactions très rapides avec le radical $\mathrm{DPPH}^{\bullet}$, la totalité de l'activité antiradicalaire peut être attribuée à la vitamine C. Dans le cas de réactions plus lentes, en plus de la vitamine C, ce sont les polyphénols (citroflavonoïdes) qui contribuent à l'activité antioxydante totale. Les extraits méthanoliques des peaux fraîches (flavédo) contiennent plus de vitamine C que les extraits de peaux sèches. Conclusion. Les peaux d'oranges constituent d'immenses sources d'antioxydants qui peuvent trouver leur application dans différents domaines : agroalimentaire, cosmétique, pharmaceutique et autres.
\end{abstract}

Tunisie / Citrus sinensis / fruits / jus d'orange / pelure / utilisation des déchets / antioxydant naturel / acide ascorbique 


\section{Introduction}

De nombreuses études épidémiologiques ont mis en évidence le lien entre un régime riche en fruits et légumes et une diminution du risque de maladies cardiovasculaires et de certaines formes de cancers [1-6]. Des études ont montré que ce sont les antioxydants présents dans ces fruits et légumes qui participent aux effets protecteurs [7, 8].

Les polyphénols sont les antioxydants les plus abondants dans nos régimes alimentaires [9-12]. Ils constituent un ensemble de molécules très répandues dans le règne végétal [13-15], en particulier chez les plantes supérieures où ils se retrouvent dans les racines, les feuilles, les fleurs et les fruits.

Depuis quelques années de nombreuses recherches ont été consacrées aux polyphénols et à leur rôle bénéfique pour la santé humaine. Les polyphénols sont des agents réducteurs [16-18] comme d'autres composés tels que la vitamine $\mathrm{C}$, la vitamine $\mathrm{E}$ et les caroténoïdes. Ces puissants antioxydants sont capables de piéger les radicaux libres générés en permanence par notre organisme ou formés en réponse à des agressions extérieures (pollutions diverses, infections, etc.) [19-21]. Les polyphénols peuvent renforcer nos défenses naturelles contre le stress antioxydant en protégeant les constituants tissulaires [16, 22-25]. Ils peuvent également agir comme chélateurs d'ions métalliques pro-oxydants, comme le fer et le cuivre, et inhiber la production des radicaux libres [26-28].

Les polyphénols sont capables d'empêcher l'oxydation des lipoprotéines de faible densité (Low Density Lipoproteins ou LDL) [29] et pourraient de ce fait protéger l'organisme contre les infarctus du myocarde ou l'athérosclérose coronarienne qui sont associés aux taux élevés de cholestérol au niveau des LDL circulant dans le sang. Toutefois cette action démontrée par des tests in vitro ou ex vivo reste encore à être prouvée in vivo chez l'homme. Les polyphénols peuvent également jouer un rôle d'antimutagène, d'anti-inflammatoire, d'antimicrobien, d'antitumorale ou d'antivirale [7, 22, 30-34].

L'orange constitue une source de régime alimentaire riche en composés bioactifs. En plus de la vitamine $\mathrm{C}$ et $\mathrm{E}$ et des caroténoïdes, l'orange contient des composés polyphénoliques [35, 36] qui regroupent les flavonoïdes et plus précisément des flavanones et des flavones glycosylées et polymethoxylées. Malterud et ses collaborateurs ont pu isoler neuf flavanones des peaux d'oranges, l'hésperidine étant la flavanone majoritaire [37].

La production annuelle d'oranges en Tunisie a varié de $(210$ à 240$) \times 10^{3}$ t entre 1995 et 2003. Près de la moitié de cette production a concerné la variété Maltaise [38]. L'industrie de l'orange tunisienne étant orientée vers la production de jus et de nectar d'oranges, de très gros tonnages de déchets sont produits.

Afin d'évaluer la possibilité de recycler les déchets issus de l'industrie de l'orange tunisienne, nous avons mesuré l'activité antiradicalaire des fruits de cinq variétés d'oranges par une méthode spectrophotométrique impliquant le radical $\mathrm{DPPH}^{\bullet}$ en milieu méthanolique [39, 40]. Puis nous avons évalué les teneurs en vitamine $\mathrm{C}$ des fruits des variétés étudiées.

\section{Matériel et méthodes}

\subsection{Solvants et réactifs}

Au cours de nos expérimentations, l'eau permutée et le méthanol ont été utilisés comme solvants d'extraction.

L'eau permutée a été préparée à partir d'eau osmosée, filtrée et purifiée sur deux types de colonnes : deux colonnes échangeuses d'ions (Bioblock Scientific R3-83002 et M3-83006) et une colonne à base de charbon actif (Bioblock Scientific ORC-83005).

Le méthanol de qualité spectroscopique (Merck, SeccoSolv ${ }^{\circledR}$, p.a.) a été longuement dégazé avec de l'argon par passage préalable de ce gaz sur une cartouche Oxiclear (Labclear, DGP-250-R1) afin d'éliminer toute trace d'oxygène. Le radical $\mathrm{DPPH}^{\bullet}$ $\left(\mathrm{C}_{18} \mathrm{H}_{12} \mathrm{~N}_{5} \mathrm{O}_{6}, \mathrm{M}=394,3 \mathrm{~g} \cdot \mathrm{mol}^{-1}\right.$, Sigma, France, $90 \%$ ) et l'acide $\mathrm{L}(+)$ ascorbique $\left(\mathrm{C}_{6} \mathrm{H}_{8} \mathrm{O}_{6}, \mathrm{M}=176,13 \mathrm{~g} \cdot \mathrm{mol}^{-1}\right.$, Merck, Allemagne) ont été utilisés sans purification ultérieure. 
Tableau I.

Pourcentage en matière sèche des peaux d'oranges des différentes variétés d'orangers, étudiées pour mesurer leur activité radiculaire par le radical DPPH (Tunisie, valeur moyenne pour dix oranges).

\begin{tabular}{lccccc} 
Variété & $\begin{array}{c}\text { Masse } \\
\text { du fruit } \\
(\mathrm{g})\end{array}$ & $\begin{array}{c}\text { Masse } \\
\text { de peaux fraîches } \\
(\mathrm{g})\end{array}$ & $\begin{array}{c}\text { Masse de peaux } \\
\text { par rapport à la masse d'un fruit } \\
(\%)\end{array}$ & $\begin{array}{c}\text { \% matière sèche } \\
\text { dans les peaux fraîches }\end{array}$ & $\begin{array}{c}\% \text { matière sèche } \\
\text { dans les peaux sèches }\end{array}$ \\
\hline Chemi & 206,5 & 69,62 & 33,71 & 27,0 & 83,0 \\
Maltaise 1 & 188,5 & 70,63 & 37,47 & 25,4 & 81,0 \\
Maltaise 2 & 181,5 & 67,5 & 37,19 & 27,0 & 83,0 \\
Orange douce & 177,0 & 83,0 & 46,89 & 26,0 & 80,8 \\
Thomson & 223,5 & 98,0 & 43,84 & 26,0 & 73,0
\end{tabular}

\subsection{Matériel végétal et échantillonnage}

Nos travaux ont porté sur l'étude des propriétés du jus des fruits de cinq variétés d'orangers [Citrus sinensis (L.) Osbeck] couramment exploitées en Tunisie :

- deux variétés de Maltaise: Maltaise 1, orange destinée à la fabrication du jus, et Maltaise 2, orange de bouche,

- trois autres variétés rencontrées en vergers tunisiens : Chemi, orange douce et Thomson

Les fruits ont été cueillis à maturation. Pour effectuer nos mesures, dix oranges de chacune des variétés étudiées, de masses comprises entre (110 et 220) g (tableau I), ont été lavées à l'eau permutée, sectionnées en deux et rapidement pressées à la main. Le jus d'orange frais ( $400 \mathrm{~mL}$ pour chaque variété) a été stocké à $-18{ }^{\circ} \mathrm{C}$ jusqu'à utilisation.

\subsection{Préparation des extraits de jus}

Au moment des dosages, le jus d'orange a été centrifugé deux fois de suite à la vitesse de 1500 tours $\cdot \mathrm{min}^{-1}$ pendant 10 min à l'aide d'une centrifugeuse Beckman Microfuge ETM. La solution limpide obtenue a été utilisée sans purification ultérieure.

\subsection{Préparation des extraits des peaux sèches et fraîches d'orange}

La procédure utilisée pour extraire les composés phénoliques des peaux fraîches et sèches des oranges a été empruntée à la littérature [41]. Les peaux, dont l'albédo, ont été séparées des oranges fraîches. La moitié des peaux fraîches (environ $400 \mathrm{~g}$ ) a été séchée en étuve à $30-40{ }^{\circ} \mathrm{C}$ pendant 3 jours jusqu'à obtention de $25 \%$ du poids des peaux de départ. Ces peaux séchées et les peaux conservées fraîches ont été broyées finement à l'aide d'un mixeur électrique (Mixeur Minipimer professional MR $5550 \mathrm{M}$ CA, Braun).

Pour préparer les extraits analysés, $50 \mathrm{~mL}$ d'eau à $+4{ }^{\circ} \mathrm{C}, 50 \mathrm{~mL}$ d'eau à $+90^{\circ} \mathrm{C}$ et $60 \mathrm{~mL}$ de méthanol ont été ajoutés successivement à une prise d'essai de $5 \mathrm{~g}$ de peaux sèches (environ 20 \% d'eau) ou de peaux fraîches (environ $75 \%$ d'eau et de volatils) de chacune des variétés d'orangers testées. Après agitation vigoureuse de quelques minutes, les mélanges ont été laissés au repos pendant 3 jours à $+4{ }^{\circ} \mathrm{C}$. Avant chaque analyse, les solutions ont été centrifugées deux fois de suite à la vitesse de 1500 tours $\cdot \mathrm{min}^{-1}$ pendant 10 min. La solution surnageante limpide a été utilisée telle quelle sans purification ultérieure.

\subsection{Préparation des solutions d'acide ascorbique et de DPPH}

Une solution d'acide ascorbique de concentration voisine de $8 \cdot 10^{-4} \mathrm{~mol} \cdot \mathrm{L}^{-1}$ ainsi qu'une solution de DPPH de concentration de l'ordre de $10^{-4} \mathrm{~mol} \cdot \mathrm{L}^{-1}$ ont été préparées dans du méthanol préalablement dégazé par de l'argon. La solution du DPPH a été conservée à l'abri de la lumière et à $+4{ }^{\circ} \mathrm{C}$. La concentration analytique a été déterminée par spectrophotométrie $\left(\varepsilon^{515}=\right.$ $12500 \mathrm{M}^{-1} \cdot \mathrm{cm}^{-1}$, figure 1) [39]. 
Figure 1.

Spectres d'absorption d'une solution de $\mathrm{DPPH}^{\bullet}$ à $9,93 \cdot 10^{-5} \mathrm{~mol} \cdot \mathrm{L}^{-1}$ dans le méthanol, $\left[(25,0 \pm 0,2)^{\circ} \mathrm{C}\right]$.
Figure 2.

Mesure de l'absorbance à $515 \mathrm{~nm}$ du radical $\mathrm{DPPH}^{\bullet}$ à $9,93 \cdot 10^{-5} \mathrm{~mol} \cdot \mathrm{L}^{-1}$, en fonction du temps, pour des volumes d'extraits de peaux séchées d'oranges de variété Maltaise [solvant : méthanol ; $(25,0 \pm$ $\left.0,2){ }^{\circ} \mathrm{C} ; l=1 \mathrm{~cm}\right]$.
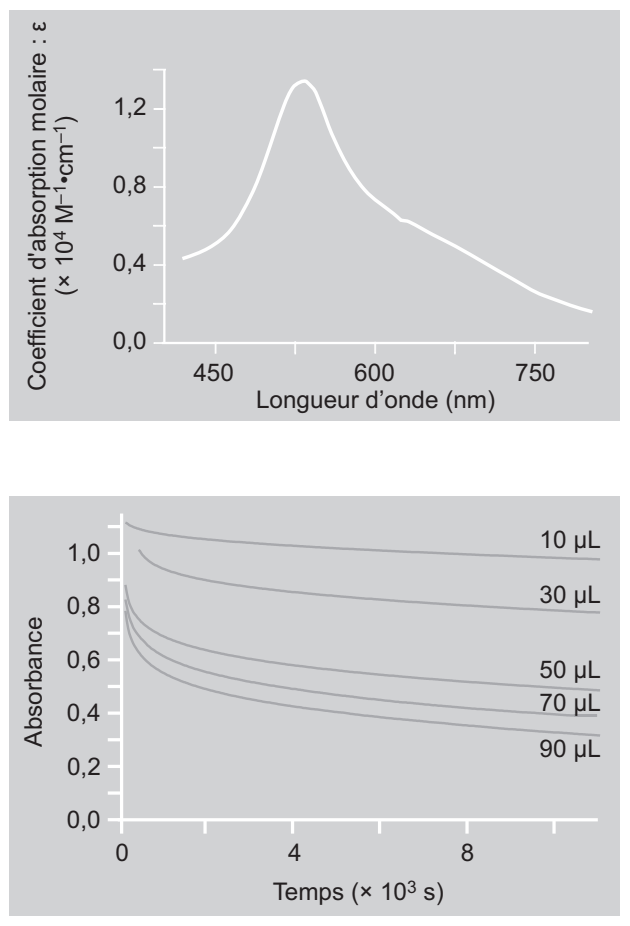

\subsection{Mesure du pH}

Le $\mathrm{pH}$ a été mesuré au moyen d'une microélectrode de verre combinée (Metrohm 6.0234.500, Long Life) et d'un millivoltmètre (Tacussel ISIS 2000). L'électrode de référence $\mathrm{Ag} / \mathrm{AgCl}$ a été remplie par une solution de $\mathrm{NaCl}$ 0,1 M (Fluka, p.a.).

\subsection{Mesure de l'activité antioxydante}

Le pouvoir antiradicalaire des jus d'orange ou des extraits de peaux fraîches ou sèches a été évalué en suivant la disparition, en fonction du temps, du radical $\mathrm{DPPH}^{\bullet}$ par spectrophotométrie d'absorption UV-visible à $515 \mathrm{~nm}$. Le nombre de radicaux libres réduits par une molécule d'antioxydant a été calculé après établissement de l'état stationnaire de la réaction.

Afin de déterminer la concentration analytique de la solution de DPPH, 2 mL ont été introduits dans une cellule en quartz (Hellma) de trajet optique $1 \mathrm{~cm}$, thermostatée à (25,0 \pm $0,2){ }^{\circ} \mathrm{C}$ (thermostat Haake FJ). Un spectre d'absorption UV-visible a été enregistré de
(400 à 800) nm au moyen d'un spectrophotomètre Kontron (Uvikon 941) (figure 1).

D'après la loi de Beer-Lambert :

$(\mathrm{DPPH})_{\mathrm{t}}=\left(\mathrm{A}_{\mathrm{t}}^{515} / l \times \varepsilon^{515}\right)$ (équation 1$)$,

où $(\mathrm{DPPH})_{\mathrm{t}}$ est la concentration molaire du radical $\mathrm{DPPH}^{\bullet}$ dans le méthanol à un instant $t ; \varepsilon^{515}$ est le coefficient d'absorption molaire à $515 \mathrm{~nm}$ et égal à $12500 \mathrm{~mol} \cdot \mathrm{L}^{-1} \cdot \mathrm{cm}^{-1} ; l$ est le trajet optique mesuré en $\mathrm{cm}$ et $\mathrm{A}_{\mathrm{t}}^{515}$ est l'absorbance de la solution de $\mathrm{DPPH}^{\bullet}$ à $515 \mathrm{~nm}$.

À l'aide d'une microburette à piston (Eppendorf), nous avons ajouté $2 \mathrm{~mL}$ de DPPH à différents volumes d'échantillon : échantillons de (2 à 20) $\mu \mathrm{L}$ pour les jus et de (10 à 100) $\mu \mathrm{L}$ pour les extraits des peaux d'orange. Le mélange a été rapidement agité avant mesure du déclin de l'absorbance à 515 nm en fonction du temps, jusqu'à obtention d'un état stationnaire.

Pour mettre en évidence la contribution des divers composés phénoliques dans l'activité antioxydante des extraits, nous avons mesuré, par spectrophotomètrie UVvisible, la diminution de l'absorbance en fonction du temps du radical $\mathrm{DPPH}^{\bullet}$ réagissant avec différents volumes d'extraits de peaux dans une gamme comprise entre (10 et 90) $\mu \mathrm{L}$, jusqu'à obtention d'un état stationnaire. Cet état stationnaire a été atteint au bout d'environ $9000 \mathrm{~s}$ (figure 2). Toutes les séries d'expériences ont été reproduites trois fois et les résultats sont donnés avec un intervalle de confiance de $95 \%(3 \sigma)$.

\subsection{Calcul de l'activité antioxydante}

En présence d'un antioxydant, le DPPH ${ }^{\bullet}$ se transforme plus au moins rapidement en diphénylpicrylhydrazine $\left(\mathrm{DPPH}_{2}\right)$, faiblement colorée en jaune par captation d'un hydrogène de l'antioxydant (figure 3).

La concentration efficace $\mathrm{CE}_{50}$ correspond à la concentration en antioxydant pour laquelle la diminution d'absorbance à l'état stationnaire atteint $50 \%$ de l'absorbance initiale d'une solution de $\mathrm{DPPH}^{\bullet}$ de même concentration mais sans antioxydant [39]. La valeur de $\mathrm{CE}_{50}$, exprimée en $\mathrm{mol} \cdot \mathrm{L}^{-1}$, a été déterminée graphiquement à partir de plusieurs mesures expérimentales du pourcentage de $\mathrm{DPPH}^{\bullet}$ restant, pour diverses 
concentrations en antioxydant une fois l'état stationnaire atteint.

Le pourcentage de $\mathrm{DPPH}^{\bullet}$ restant après la réaction radicalaire conduite avec un volume $V$ d'antioxydant a été déterminé par l'expression suivante [39] :

$\% \mathrm{DPPH}^{\bullet}=\left[\left(\mathrm{A}_{\infty} / \mathrm{A}_{\infty}^{0}\right) \times 100\right]($ équation 2$)$.

$\mathrm{A}_{\infty}^{0}$ est l'absorbance d'une solution témoin de $\mathrm{DPPH}^{\bullet}$ et A est l'absorbance finale d'une solution de $\mathrm{DPPH}^{\bullet}$ en présence d'un volume $V$ d'antioxydant.

La concentration efficace ou concentration analytique d'antioxydant CE est défini par :

$$
\mathrm{CE}=\left[(\mathrm{AH})_{0} \times\left(V / V_{t o t}\right)\right] \text { (équation 3). }
$$

$(\mathrm{AH})_{0}$ est la concentration initiale en antioxydant ; $V_{\text {tot }}$ est le volume total tel que $V_{t o t}=V_{1}+V ; V_{1}$ est le volume de la solution de $\mathrm{DPPH}^{\bullet}$ et $V$ est le volume de la solution de l'antioxydant ajouté ( $V$ très inférieur à $V_{1}$ car $V \approx 10^{-1} V_{1}$ ).

Nous avons calculé, pour la vitamine $C$, le pourcentage de DPPH restant ainsi que la concentration efficace. Le pourcentage du DPPH et la CE ont été calculés d'après les équations 2 et 3 (figure 4 ).

Nous avons également déterminé la puissance antiradicalaire PAR (en moles de $\mathrm{DPPH}^{\bullet}$ par moles d'antioxydant) de la vitamine $\mathrm{C}$, mesurée par :

$$
\operatorname{PAR}=\left[\left(\mathrm{DPPH}^{\bullet}\right)_{\mathrm{tot}} / \mathrm{CE}_{50}\right](\text { équation } 4) \text {. }
$$

Dans des conditions expérimentales identiques de concentration en radical $\mathrm{DPPH}^{\bullet}$, nous avons mesuré un $\mathrm{V}_{50}$ de vitamine $\mathrm{C}$ d'une solution d'acide ascorbique de concentration connue $\left[(\text { Vit } \mathrm{C})_{0} \approx 10^{-4} \mathrm{~mol} \cdot \mathrm{L}^{-1}\right]$ et un $\mathrm{V}_{50}$ d'un extrait de jus (figure 5) ou de peau de fruit de concentration en vitamine $\mathrm{C}$ inconnue. La détermination de ces deux données et le calcul du nombre de moles $n_{50}$ d'une solution de vitamine $\mathrm{C}$ de concentration connue nécessaire pour inhiber $50 \%$ des radicaux DPPH ${ }^{\bullet}$ nous a permis d'estimer la concentration molaire et massique de vitamine $\mathrm{C}$ équivalente dans les échantillons selon les équations 5 à 7 :

$$
\left.n_{50}=\mathrm{V}_{50}(\text { vit C }) \times(\text { vit C })_{0} \text { (équation } 5\right) \text {, }
$$

(vit C) équiv molaire dans le jus de fruit $=n_{50} /$ $\mathrm{V}_{50}$ du jus (équation 6)

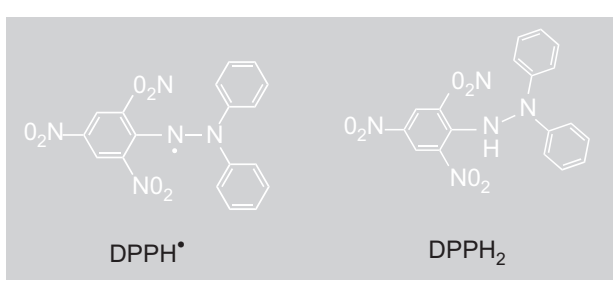

Figure 3.

Structures chimiques du 1,1-diphényl-2-picrylhydrazyl $\left(\mathrm{DPPH}^{\circ}\right)$ et de sa forme réduite, le 2,2-diphényl-1-

picrylhydrazine $\left(\mathrm{DPPH}_{2}\right)$.

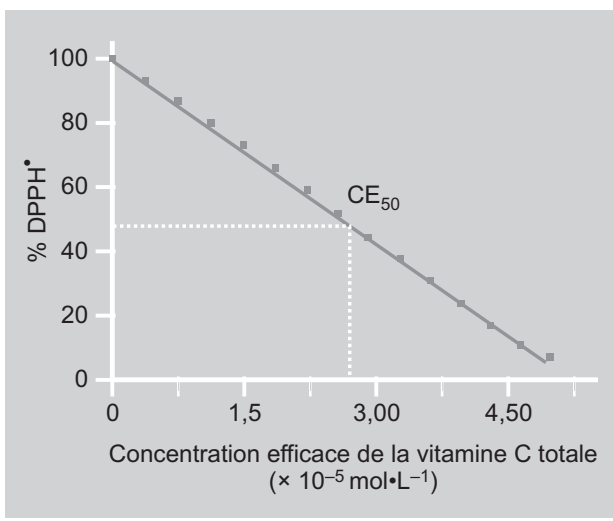

Figure 4.

Pourcentage de $\mathrm{DPPH}^{\circ}$ consommé en fonction de la concentration efficace $\mathrm{CE}$ de la vitamine $\mathrm{C}$ [solvant : méthanol ; $\left(\mathrm{DPPH}^{\circ}\right)_{\text {tot }}=1,04 \cdot 10^{-5} \mathrm{M}$; $\left.(25,0 \pm 0,2){ }^{\circ} \mathrm{C}\right]$

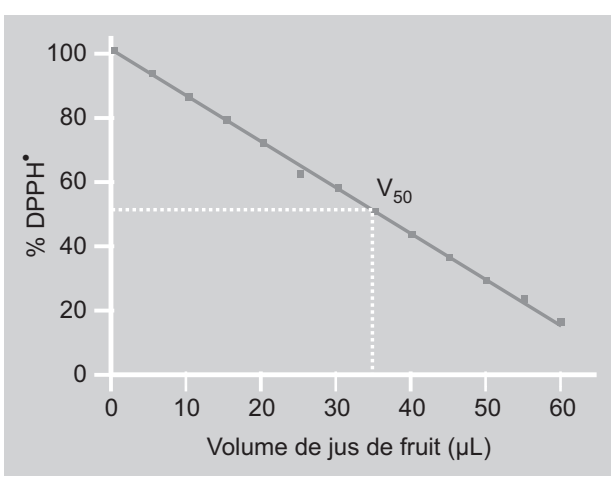

Figure 5.

Pourcentage de DPPH consommé en fonction du volume de jus d'orange ajouté. Extrait préparé à partir de la variété Orange douce [solvant : méthanol ; $\left(\mathrm{DPPH}^{\circ}\right)_{\text {tot }}=$ $\left.9,38 \cdot 10^{-5} \mathrm{M} ;(25,0 \pm 0,2)^{\circ} \mathrm{C}\right]$. (vit C) équiv massique dans le jus de fruit = (vit C) équiv molaire $\times \mathrm{M}_{\mathrm{vitam} \mathrm{C}}$ (équation 7 ),

où $\mathrm{M}_{\text {vitam } \mathrm{C}}$ est la masse molaire de la vitamine $\mathrm{C}$.

(Vit C) $)_{0}$ est la concentration initiale d'une solution de vitamine $\mathrm{C}$ de concentration connue $\left[(\text { vit } \mathrm{C})_{0} \approx 8 \cdot 10^{-4} \mathrm{~mol} \cdot \mathrm{L}^{-1}\right] ; \mathrm{V}_{50}$ (vit C) est le volume en $\mu \mathrm{L}$ de cette solution de vitamine $\mathrm{C}$ nécessaire pour réduire $50 \%$ des radicaux $\mathrm{DPPH}^{\bullet} ; n_{50}$ est le nombre de moles de la solution de vitamine $\mathrm{C}$ de concentration (vit $\mathrm{C})_{0}$ nécessaire pour réduire $50 \%$ des radicaux $\mathrm{DPPH}^{\bullet} ; \mathrm{V}_{50}$ du jus est le volume $(\mu \mathrm{L})$ d'un extrait d'oranges (jus, extrait de peaux fraîches ou sèches) de concentration inconnue nécessaire pour réduire $50 \%$ des 


\section{Tableau II.}

$\mathrm{pH}$ mesuré dans les différents jus d'oranges extraits des fruits de cinq variétés tunisiennes étudiées pour mesurer leur activité radiculaire par le radical DPPH.

\begin{tabular}{lc} 
Variété & $\mathrm{pH}$ \\
\hline Chemi & 3,54 \\
Maltaise 1 & 3,36 \\
Maltaise 2 & 3,32 \\
Orange douce & 4,20 \\
Thomson & 4,10
\end{tabular}

\section{Tableau III.}

Teneur en vitamine $\mathrm{C}$ en équivalent molaire ou massique dans différents extraits de jus provenant de cinq variétés tunisiennes étudiées pour mesurer leur activité radiculaire par le radical DPPH.

\begin{tabular}{lcc} 
Variété & $\begin{array}{c}\text { Vitamine }]_{\text {équiv molaire }} \\
\times 10^{-3}\left(\mathrm{~mol}^{-1}\right) 3 \sigma\end{array}$ & $\begin{array}{c}\text { Vitamine } \mathrm{C}]_{\text {équiv massique }} \\
\left(\mathrm{mg} \cdot \mathrm{L}^{-1}\right) 3 \sigma\end{array}$ \\
\hline Chemi & 2,70 & $476 \pm 96$ \\
Maltaise 1 & 2,65 & $466 \pm 34$ \\
Maltaise 2 & 2,22 & $390 \pm 10$ \\
Orange douce & 1,51 & $267 \pm 112$ \\
Thomson & 2,71 & $478 \pm 160$
\end{tabular}

Tableau IV.

Teneur en vitamine $C$ (en équivalent massique) dans différents extraits de peaux fraîches et sèches provenant de cinq variétés tunisiennes étudiées pour mesurer leur activité radiculaire par le radical $\mathrm{DPPH}$.

\begin{tabular}{lcc} 
Variété & Peaux fraîches & Peaux sèches \\
\hline Chemi & {$\left[\mathrm{mg} \cdot 100 \mathrm{~g}^{-1}\right.$ matière sèche $\left.(3 \sigma)\right]$} \\
Maltaise 1 & $209 \pm 9$ & $195 \pm 55$ \\
Maltaise 2 & $240 \pm 55$ & $204 \pm 34$ \\
Orange douce & $191 \pm 29$ & $170 \pm 34$ \\
Thomson & $236 \pm 46$ & $223 \pm 15$ \\
& $161 \pm 24$ & $146 \pm 101$
\end{tabular}

radicaux $\mathrm{DPPH}^{\bullet}$; (vit C) équiv molaire est la concentration en vitamine $\mathrm{C}$ équivalente (en $\mathrm{mol} \cdot \mathrm{L}^{-1}$ ) dans les extraits d'oranges; (vit C) équiv massique est la concentration en vitamine $\mathrm{C}$ équivalente $\left(\mathrm{mg} \cdot \mathrm{L}^{-1}\right)$ dans les extraits d'oranges.

\subsection{Traitement des données}

Pour déterminer la concentration efficace à 50 \% pour la vitamine C, les données ont été traitées par une méthode des moindres carrés linéaires (MicrocalTM OriginTM version 5.0) [42].

\section{Résultats et discussion}

Pour les différentes solutions d'acide ascorbique testées, nous avons vérifié que la puissance antiradicalaire PAR était égale à 3,8 \pm 0,7 moles de $\mathrm{DPPH}^{\bullet}$ par moles d'antioxydant. Cette valeur nous a permis de comparer l'efficacité des divers substrats analysés.

La vitamine $\mathrm{C}$ est majoritairement présente sous forme d'acide ascorbique dans les différents extraits d'oranges pour lesquels le $\mathrm{pH}$ mesuré a varié de 3,32 à 4,20 selon la variété (tableau II). Dans cette zone de $\mathrm{pH}$ acide, les antioxydants (vitamine $\mathrm{C}$ et citroflavonoïdes) sont présents sous forme protonée.

Pour les différentes variétés d'orange étudiées, les teneurs équivalentes de vitamine C des jus ont été comprises entre (267 et 478) $\mathrm{mg} \cdot \mathrm{L}^{-1}$ (tableau III). Dans le domaine d'incertitude donné, les taux de vitamine $\mathrm{C}$ des oranges douces et Thomson se recouvrent, néanmoins les oranges douces présentent les plus faibles teneurs en vitamine C (276 \pm 112) $\mathrm{mg} \cdot \mathrm{L}^{-1}$ et le $\mathrm{pH}$ le plus élevé $(\mathrm{pH}=$ 4,20).

Les extraits méthanoliques des peaux sèches ont révélé des concentrations en acide ascorbique plus faibles que ceux des peaux fraîches (tableau IV). Pour les peaux sèches, les concentrations en vitamines $\mathrm{C}$ ont varié de (170 à 223) mg pour $100 \mathrm{~g}$ de matière sèche, les oranges Maltaise 2 ayant les concentrations les plus faibles et les oranges douces, les plus fortes. Les peaux fraîches ont présenté des concentrations en vitamine C variant de $191 \mathrm{mg} \cdot 100 \mathrm{~g}^{-1}$ de matière sèche pour les oranges de la variété Maltaise 2 à $236 \mathrm{mg} \cdot 100 \mathrm{~g}^{-1}$ de matière 
sèche pour les oranges douces. Ces résultats suggèrent que les peaux perdraient une certaine quantité de vitamine $\mathrm{C}$ sous l'effet du séchage. Cela est en accord avec les résultats obtenus par Higashi-Okai et ses collaborateurs [41].

Nous avons cherché également à évaluer la contribution des citroflavonoïdes dans l'activité antiradicalaire des extraits de peaux séchées de la variété Maltaise vis-àvis du radical $\mathrm{DPPH}^{\bullet}$. Supposant que la vitamine $\mathrm{C}$ réagit avec le radical $\mathrm{DPPH}^{\bullet}$ en moins de $30 \mathrm{~s}$, nous pouvons penser que les cinétiques lentes de réactions radicalaires pourraient impliquer d'autres substrats d'origine polyphénolique. L'analyse de l'évolution dans le temps de l'absorbance d'un mélange de $\mathrm{DPPH}^{\bullet}$ avec un extrait de peaux sèches de la variété Maltaise (figure 2) nous conduit à remarquer que l'état stationnaire n'est atteint qu'au bout de plusieurs heures, tandis que la réaction est très rapide avec les jus d'orange.

Le traitement graphique issu des équations 5 à 7 nous a permis de déterminer des volumes efficaces à $50 \%$ pour les réactions rapides et lentes de réaction entre les radicaux $\mathrm{DPPH}^{\bullet}$ et les extraits méthanoliques de peaux sèches de la variété maltaise (figure O)

Nous obtenons ainsi des valeurs de $\mathrm{V}_{50}$ de l'ordre de $125 \mu \mathrm{L}$ et $40 \mu \mathrm{L}$ qui correspondent respectivement aux réactions radicalaires qui impliquent la vitamine $\mathrm{C}$ (réactions rapides) et les dérivés polyphénoliques (réactions lentes).

Ces résultats suggèrent que la vitamine $\mathrm{C}$ contenue dans les peaux d'oranges constituerait le principal antioxydant pour des réactions rapides, tandis que, pour les réactions plus lentes, des polyphénols présents en plus de la vitamine $\mathrm{C}$ interviendraient dans l'activité antiradicalaire.

\section{Conclusion}

Les jus d'orange, acides, se sont révélés contenir de la vitamine $\mathrm{C}$ dans des proportions de l'ordre de $400 \mathrm{mg} \cdot \mathrm{L}^{-1}$. Les peaux d'oranges, quant à elles, renferment, dans leurs parties colorées apparentes (flavedo), de plus faibles quantités de vitamine $\mathrm{C}$, ainsi

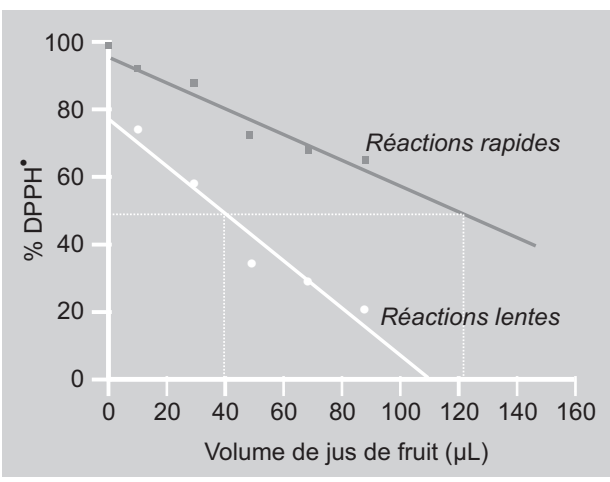

Figure 6.

Pourcentage de DPPH consommé en fonction du volume de jus d'orange ajouté. Extrait préparé à partir de la variété Maltaise [solvant : méthanol ; $\left(\mathrm{DPPH}^{*}\right)_{\text {tot }}=$ $\left.9,93 \cdot 10^{-5} \mathrm{M} ;(25,0 \pm 0,2)^{\circ} \mathrm{C}\right]$.

que d'autres antioxydants, des polyphénols, en particulier. Ces antioxydants se caractérisent par des vitesses de réaction radicalaire très différentes en présence du radical $\mathrm{DPPH}^{\bullet}$. En effet, la totalité de l'activité antiradicalaire serait due à la vitamine $\mathrm{C}$ dans le cas de réactions très rapides, tandis que, dans les réactions à cinétiques lentes, ce serait les polyphénols qui contribueraient en grande partie à l'activité antioxydante totale. Ces résultats, qui sont en accord avec de nombreuses études issues de la littérature [41], montrent la forte potentialité industrielle du recyclage de déchets d'oranges en tant qu'antioxydants alimentaires.

\section{Références}

[1] Steinmetz K.A., Potter J.D., Vegetables, fruit and cancer prevention: a review, J. Am. Diet. Assoc. 53 (1996) 536-543.

[2] Liu S., Lee I.M., Ajani U., Intake of vegetables rich in carotenoids and risk of coronary heart disease in men: the physicians' health study, Int. J. Epidemiol. 30 (2001) 130-135.

[3] Joshipura K.J., Hu F.B., Manson J.E., The effect of fruit and vegetable intake on risk for coronary heart disease, Ann. Intern. Med. 134 (2001) 1106-1114.

[4] Gerber M., Boutron-Ruault M.C., Hercberg S., Riboli E., Scalbert A., Siess M.H., Food and cancer: state of the art about the protective effect of fruits and vegetables, Bull. Cancer 89 (2002) 293-312.

[5] Sasazuki S., Case-control study of nonfatal myocardial infarction in relation to selected foods in Japanese men and women, Jpn. Circ. J. 65 (2001) 200-206.

[6] Bazzano L.A., He J., Ogden L.G., Fruit and vegetable intake and risk of cardiovascular 
disease in US adults: the first national health and nutrition examination survey epidemiologic follow-up study, Am. J. Clin. Nutr. 76 (2002) 93-99.

[7] Hertog M.G.L., Feskens E.J.M., Hollman P.C.H., Katan J.B., Kromhout D., Dietary antioxidant flavonoids and risk of coronary heart disease: the Zutphen elderly study, Lancet 342 (1993) 1007-1011.

[8] Gee J.M., Johnson I.T., Polyphenolic compounds: interactions with the gut and implications for human health, Curr. Med. Chem. 8 (2001) 1245-1255.

[9] Kahkonen M.P., Hopia A.I., Vuorela H.J., Rauha J.P., Pihlaja K., Kujala T.S., Heinonen M., Antioxidant activity of plant extracts containing phenolic compounds, J. Agric. Food Chem. 47 (1999) 3954-3962.

[10] Scalbert A., Williamson G., Dietary intake and bioavailability of polyphenols, J. Nutr. 130 (2000) 2073S-2085S.

[11] Scalbert A., Morand C., Manach C., Rémésy C., Absorption and metabolism of polyphenols in the gut and impact on health, Biomed. Pharmacother. 56 (2002) 276-282.

[12] Sakakibara H., Honda Y., Nakagawa S., Ashida H., Kanazawa K. , Simutaneous determination of all polyphenols in vegetables, fruits and teas, J. Agric. Food Chem. 51 (2003) 571-581.

[13] Mompon B., Lemaire B., Mengal P., Surbled M., Extraction des polyphénols : du laboratoire à la production industrielle, in : Vercauteren J., Chèze C., Triaud J. (Eds.), Polyphénols 96, Inra, Paris, France, 1998, pp. 31-43.

[14] Bravo L., Polyphenols: chemistry, dietary sources, metabolism, and nutritional significance, Nutr. Rev. 56 (1998) 317-333.

[15] Harborne J.B., Williams C.A., Advances in flavonoid research since 1992, Phytochemistry 55 (2000) 481-504.

[16] Acworth I.N., Bailey B., The handbook of oxidative metabolism, Ed. ESA Inc, Chelmsford, 1995, 37 p.

[17] Rice-Evans C.A., Miller N.J., Paganga G., Structure-antioxidant activity relationships of flavonoids and phenolic acids, Free Radic. Biol. Med. 20 (1996) 933-956.

[18] Silva F.A.M., Borges F., Guimaraes C., Lima J.L.F.C., Matos C., Reis S., Phenolic acids and derivatives: studies on the relationship among structure radical scavenging activity, and physicochemical parameters, J. Agric. Food Chem. 48 (2000) 2122-2126.

[19] Lachance P.A., Nakat Z., Jeong W.S., Antioxidants: an integrative approach, Nutrition 17 (2001) 835-838.

[20] Fang Y.Z., Yang S., Wu G., Free radicals, antioxidants, and nutrition, Nutrition 18 (2002) 872-879.

[21] Wilson A., Salamatian L., Les radicaux libres : une question d'équilibre, Univ. Versailles/ Saint-Quentin-en-Yvelines, Rapp. DESS IST, Versailles, France, 2002-2003, 37 p.

[22] Salah N., Miller N.J., Paganga G., Tijburg L., Bolwell G.P., Rice-Evans C., Polyphenolic flavanols as scavengers of aqueous phase radicals and as chain-breaking antioxidants, Arch. Biomed. Biophys. 322 (2) (1995) 339346.

[23] Halliwell B., Antioxidants in human health and disease, Ann. Rev. Nutr. 16 (1996) 33-50.

[24] Deng W., Fang X., Wu J., Flavonoids function as antioxidants: by scavenging reactive oxygen species or by chelating iron, Radiat. Phys. Chem. 50 (1997) 271-276.

[25] Croft K.D., The chemistry and biological effects of flavonoids and phenolic acids, Ann. NY Acad. Sci. 854 (1998) 435-442.

[26] Geisser P., Iron therapy with special emphasis on oxidative stress, Ed. Vifor (Int.) Inc., Switzerland, 1998, 142 p.

[27] Pierre J.L., Fontecave M., Iron and activated oxygen species in biology: the basic chemistry, Biometals 12 (1999) 195-199.

[28] Fernandez M.T., Mira M.L., Florencio M.H., Jennings K.R., Iron and copper chelation by flavonoids: an electrospray mass spectrometry study, J. Inorg. Biochem. 92 (2002) 105-111.

[29] Cook N.C., Samman S., Flavonoids - chemistry, metabolism, cardioprotective effects, and dietary sources, Nutr. Biochem. 7 (1996) 66-76.

[30] Hertog M.G., Hollman P.C.H., Katan M.B., Content of potentially anticarcinogenic flavonoids of 28 vegetables and 9 fruits commonly consumed in the Netherlands, J. Agric. Food Chem. 40 (1992) 2379-2383.

[31] Halliwell B., Free radicals, antioxidants, and human disease: curiosity, cause, or consequence? Lancet 344 (1994) 721-724. 
[32] Mitscher L.M., Telikepalli H., McGhee E., Shankel D.M., Natural antimutagenic agents, Mutat. Res. 350 (1996) 142-143.

[33] Owen R.W., Giacosa A., Hull W.E., Haubner R., Spiegelhalder B., Bartsch H., The antioxidant/anticancer potential of phenolic compounds isolated from olive oil, Eur. J. Cancer 36 (2000) 1235-1247.

[34] Sala A., Recio M.D., Giner R.M., Manez S., Tournier H., Schinella G., Rios J.L., Antiinflammatory and antioxidant properties of Helichrysum italicum, J. Pharm. Pharmacol. 54 (2002) 365-371.

[35] Benavente-Garcia O., Castillo J., Marin F.R., Ortuno A., Del Rio J.A., Uses and properties of citrus flavonoids, J. Agric. Food Chem. 45 (1997) 4505-4515.

[36] Sánchez-Moreno C., Plaza L., De Ancos B., Pilar Cano M., Quantitative bioactive compounds assessment and their relative contribution to the antioxidant capacity of commercial orange juices, J. Sci. Food Agric. 83 (2003) 430-439.
[37] Malterud K.E., Rydland K.M., Inhibitors of 15lipoxygenase from orange peel, J. Agric. Food Chem. 48 (2000) 5576-5580.

[38] Anon., Rapport annuel de la banque centrale de Tunisie (2003), Tunis, Tunisie, 2004, $260 \mathrm{p}$.

[39] Brand-Williams W., Cuvelier M.E., Berset C., Use of free radical method to evaluate antioxidant activity, Lebensm. Wiss. Technol. 28 (1995) 25-30.

[40] Sánchez-Moreno C., Larrauri J.A., SauraCalixto F., A procedure to measure the antiradical efficiency of polyphenols, J. Sci. Food Agric. 76 (1998) 270-276.

[41] Higashi-Okai K., Kamimoto K., Yoshioka A., Okai Y., Potent suppressive activity of fresh and dried peels from satsuma mandarin Citrus unshiu (Marcov.) on hydroperoxide generation from oxidized linoleic acid, Phytother. Res. 16 (2002) 781-784.

[42] Anon., Microcal TM OriginTM, version 5.0, Microcal Software, Inc., Northampton, MA, USA, 1991-1997.

\section{Medida de la actividad antirradical del jugo y de las pieles de naranja tunecinas por el radical DPPH.}

Resumen - Introducción. La naranja (Citrus aurantium sinensis) es una fuente de compuestos bioactivos: vitaminas $\mathrm{C}$ y E, flavonoides (flavonas y flavanonas glicosiladas y polimetoxiladas). Para valorizar los desechos de las pieles de naranja, hemos medido la actividad antirradical del jugo y de las pieles secas y frescas de cinco variedades de naranjos de Túnez con el fin de evaluar su concentración en compuestos antioxidantes utilizables en la industria. Material y métodos. La propiedad antirradical del jugo y de los extractos de las pieles se evaluó siguiendo, en función del tiempo, la desaparición del radical estable $\mathrm{DPPH}{ }^{\bullet}$ mediante espectrofotometría de absorción UV-visible a $515 \mathrm{~nm}$. La actividad antirradical medida se experimentó en concentración eficiente $\left(\mathrm{CE}_{50}\right)$. La determinación del volumen $\mathrm{V}_{50}$ de vitamina $\mathrm{C}$ de una solución de ácido ascórbico de concentración conocida y de un volumen $\mathrm{V}_{50}$ de los extractos de jugo y de pieles secas y frescas de naranja, permitió estimar la concentración de moles y de masa de vitamina $\mathrm{C}$ equivalente en los extractos. Resultados. Los jugos de naranja contienen aproximadamente $400 \mathrm{mg} \cdot \mathrm{L}^{-1}$ de equivalente de vitamina $\mathrm{C}$. En el caso de reacciones muy rápidas con el radical $\mathrm{DPPH}$, la totalidad de la actividad antirradical puede atribuirse a la vitamina $\mathrm{C}$. En el caso de reacciones más lentas, además de la vitamina C, los polifenoles (citroflavonoides) son los que contribuyen a la actividad antioxidante total. Los extractos metilados de las pieles frescas (flavedo) contienen más vitamina C que los extractos de pieles secas. Conclusión. Las pieles de naranja constituyen unas fuentes de antioxidantes inmensas que pueden encontrar su aplicación en diferentes ámbitos: agroalimentario, cosmético, parafarmacéutico y otros.

Túnez / Citrus sinensis / frutas / zumo de naranja / piel (vegetal) / aprovechamiento de desechos / antioxidantes naturales / ácido ascórbico 